Research Paper

\title{
Salvage Carbon Ion Radiation Therapy for Locally Recurrent or Radiation-Induced Second Primary Sarcoma of the Head and Neck
}

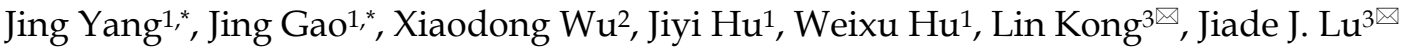 \\ 1. Department of Radiation Oncology, Shanghai Proton and Heavy Ion Center, Shanghai, China \\ 2. Division of Research and Development, Shanghai Proton and Heavy Ion Center, Shanghai, China \\ 3. Department of Radiation Oncology, Shanghai Proton and Heavy Ion Center, Fudan University Shanghai Cancer Center, Shanghai, China \\ * Both authors contributed equally to this manuscript \\ $\triangle$ Corresponding authors: Lin Kong, M.D., and Jiade J. Lu, M.D., M.B.A., Address: Shanghai Proton and Heavy Ion Center, 4365 Kangxin Road, Pudong, Shanghai, 201321, \\ China. Email: lin.kong@sphic.org.cn; jiade.lu@sphic.org.cn; Tel: +86-21-38296518 \\ (C) Ivyspring International Publisher. This is an open access article distributed under the terms of the Creative Commons Attribution (CC BY-NC) license \\ (https://creativecommons.org/licenses/by-nc/4.0/). See http://ivyspring.com/terms for full terms and conditions.
}

Received: 2017.12.12; Accepted: 2018.04.13; Published: 2018.06.04

\begin{abstract}
Purpose: Salvage radiation therapy (RT) is a potentially curative treatment option for head and neck sarcomas (HNS) that did not respond to previous treatment(s). We report the first clinical experience of carbon ion radiotherapy (CIRT) for salvage treatment of locally recurrent (LR) or RT-induced secondary HNS after surgery and/or radiotherapy.
\end{abstract}

Methods and Materials: A retrospective analysis of the ongoing prospective data registries from the Shanghai Proton and Heavy lon Center was conducted. Patients with LR-HNS who underwent surgery and/or RT and those with RT-induced second primary HNS were included. Acute and late toxicities were evaluated using the Common Terminology Criteria for Adverse Events version 4.0 and the Radiation Therapy Oncology Group late radiation toxicity scoring system, respectively. The actuarial 12-month local progression-free and overall survival rates (LPFS and OS) were calculated using the Kaplan-Meier method.

Results: Between 10/2015 and 7/2017, 19 consecutive and non-selected patients with LR-HNS or RT-induced secondary HNS received definitive doses of CIRT delivered with pencil beam scanning technology for salvage. Six patients had locally recurrent soft-tissue sarcoma, and another 6 had chondrosarcoma. Among these 12 patients, 4 had received one prior course of RT. Seven additional patients had an RT-induced second primary soft tissue sarcoma (STS)/osteosarcoma after RT. The median time between the completion of initial treatment (either surgery only or surgery followed by adjuvant RT) and salvage CIRT was 30.6 months.

The median follow-up time was 13.1 (range 1.6-41.1) months. All patients except one (for re-irradiation) completed the planned CIRT for salvage. The median dose of salvage CIRT was 60 GyE. Three patients developed local progression, and another 3 developed distant metastasis after salvage CIRT. Deaths occurred (3 patients) only in patients with radiation-induced second primary sarcoma at the time of analysis. The actuarial 12-month LPFS, DMFS and OS rates were $74.6 \%, 82.6 \%$ and $86.5 \%$, respectively.

Two patients irradiated for a second primary sarcoma had Grade 4 bleeding during CIRT, including one who experienced the rupture of an optic artery aneurysm unrelated to his disease or the salvage treatment. No patient had Grade 5 toxicity during treatment. Except for one patient who died of hemorrhage 3.5 months after the completion of CIRT, no moderate or severe late toxicities were observed.

Conclusions: With few observed acute and late toxicities, salvage CIRT can provide effective short-term tumor control. Further research, preferably in a prospective fashion, will be required to confirm the efficacy and safety of salvage CIRT in this patient population.

\section{Introduction}

Sarcoma is of mesenchymal origin and has many histological subtypes with different biological and clinical characteristics. Sarcomas of the head and neck (HNS) account for approximately 10\% of all sarcoma 
cases and less than $1 \%$ of all head and neck malignancies ${ }^{1,2,3,4,5}$. Surgery is considered the standard treatment for HNS regardless of the histological diagnosis; however, due to their proximity to the critical organs at risk (OARs), complete resection is universally difficult for locally advanced HNS. As such, the management of HNS usually requires a multidisciplinary approach with a combination of surgery, radiation therapy (RT), and depending on the histology, chemotherapy.

Despite the standard aggressive management, the prognosis of patients with HNS is the poorest compared with patients with sarcomas in other anatomical regions ${ }^{6,7}$. In patients for whom surgery is not feasible, radiation therapy provides sufficient local tumor control in only a few selected cases $8,9,10,11,12$. Not surprisingly, the outcomes in terms of local disease control and overall survival for patients with unresectable or inoperable sarcomas remains suboptima ${ }^{17,13}$ because the majority of the histological subtypes of sarcoma are relatively radio-resistant, and their proximity to the critical OARs further limits the use of high-dose RT. Furthermore, for patients who do not respond to definitive treatment for HNS, regardless of histological subtypes, or those who developed a second primary HNS after treatment (particularly radiotherapy) for a previous malignancy, the prognosis is dismal because salvage surgery or radiotherapy is usually limited by the adverse-effects caused by the prior therapy.

The physical characteristics of charged particle beams provide highly localized and precise dose distributions, which is beneficial in the treatment of tumors situated in complex anatomical environments, thus improving the therapeutic ratio. In addition, a carbon ion beam has a higher linear energy transfer (LET) and greater relative biological effectiveness (RBE) compared to photon and proton beams ${ }^{14,15}$. The synergy of both features provides a critical advantage in the management of radio-resistant malignancies that occur in close proximity to critical or sensitive OARs, such as HNS that did not respond to previous surgery and/or radiotherapy. However, to date, there is minimal evidence supporting the use of carbon ion radiation therapy (CIRT) in the setting of salvage treatment in head and neck malignancies, especially HNS 16 .

The Shanghai Proton and Heavy Ion Center (SPHIC) began routine clinical use of proton radiation therapy and intensity-modulated CIRT (IMCT) using pencil beam scanning (PBS) technology in May 2015'7. A substantial portion of this patient population have local recurrences after prior definitive treatment of their head and neck neoplasm and specifically seek salvage treatment at SPHIC. In this article, we report our clinical results of the use of IMCT at definitive doses for locally recurrent or RT-induced secondary primary HNS that did not respond to prior surgery and/or radiation therapy.

\section{Methods and Materials}

\section{Pretreatment evaluation}

The patient evaluation before salvage CIRT included a complete history and physical examination (H\&P), complete blood count and electrolyte panel, renal and liver function tests, MRI of the head and neck region (CT was allowed when MRI was contraindicated), positron emission tomography (PET), and direct or fiberoptic endoscopy when appropriate. All cases were discussed in the multidisciplinary tumor (MDT) clinic of SPHIC prior to registration and immobilization to verify the indications of their treatment. Chemotherapy was used at the discretion of the medical oncologist and was usually delivered prior to the referral of the patients. RT-induced sarcomas were defined by tumors that had occurred within the previous RT-treatment target volumes after a period of $>5$ years from the completion of previous RT for any diagnosis other than sarcoma.

\section{Intensity-modulated carbon ion radiation therapy}

The planning and treatment techniques of IMCT at SPHIC have been previously described ${ }^{18}$. Briefly, all patients were registered and immobilized in the supine position with individualized thermoplastic masks. Planning CT scans without intravenous contrast from the vertex to the inferior margin of the clavicular heads were performed at $1.5-\mathrm{mm}$ slice thickness. MRI-CT fusion was performed for all patients prior to target volume delineation. The gross tumor volume (GTV) consisted of the gross tumor discovered on clinical examination or imaging studies. We defined CTV-G as a GTV with a 1-3 mm margin to deliver the prescribed dose to the tumor. For patients who received surgery and/or chemotherapy for their recurrent disease, the pretreatment tumor bed was defined as the CTV. A maximum of a $5 \mathrm{~mm}$ margin was typically added to the CTV for the planning target volume (PTV) to mitigate potential setup errors. For 6 of the 11 patients who did not respond to previous radiotherapy, the old RT plan was obtained and the doses to the organs at risk (OARs) were identified. Recovery from previous radiation therapy dose was set at $70 \%$ regardless of the latent time between the two courses of $\mathrm{RT}^{19}$. The RT plans for 5 patients who developed a 
second primary sarcoma after their prior RT for nasopharyngeal cancer, chordoma, or pituitary adenoma were not obtained due to the extensive latent period (> 10 years). Doses were measured by Gy-equivalents (GyE) to account for the RBE differences of CIRT compared to photon-based RT. The dose constraints of the OARs were based on the TD5/5 described by Emami except for the optic nerve $\left(\mathrm{D}_{20}<30 \mathrm{GyE}\right)$, brain stem $\left(\mathrm{D}_{\max }<45 \mathrm{GyE}\right)$, spinal cord $\left(\mathrm{D}_{\max }<30 \mathrm{GyE}\right)$, and temporal lobes $\left(\mathrm{V}_{40}<7.66 \mathrm{cc}\right.$; $\mathrm{V}_{50}<4.66 \mathrm{cc}$ ), which were based on previous experience from the National Institute or Radiation Science of Japan ${ }^{20}$. Treatment planning for CIRT was performed using the Siemens Syngo ${ }^{\circledR}$ treatment planning system (version VC11).

IMCT was delivered with PBS technology. The beam arrangement varied depending on the target volume geometry and dose limits to neighboring organs at risk, such as those with prior radiation exposure. Treatments typically consisted of 2-3 beams with a median of 3 fields. Individual factors such as patient positioning reproducibility and/or beam angles were chosen for optimal dosimetry. For patients with metal dental fillings, beams were delivered from directions that avoided the hardware. Setup accuracy was confirmed with daily orthogonal X-ray using bony landmarks as a reference. Verification CT scans were typically performed on a weekly basis after the second week of the IMCT course to assess any changes in anatomy. A typical treatment plan (a patient with a local recurrence in the buccal region) is shown in Figure 1.

\section{Follow-up}

All patients were required to adhere to the standardized follow-up protocol of SPHIC. The first follow-up was within $4 \sim 6$ weeks after the completion of their particle radiation therapy (PRT), every 3 months in the first 2 years, every 6 months in the following 3 years, and annually thereafter. A complete H\&P examination focused on the head and neck regions and MRI scan of the head and neck area are required at each follow-up session. PET-CT and other laboratory or imaging studies were ordered based on any evidence of metastasis or other concurrent diseases.

\section{Data analysis}

The duration of survival was calculated from the diagnosis of the current condition, i.e., local recurrence or a second primary tumor, until death or the date of the last follow-up. The time to local, regional, or distant failure or progression was measured from the date of any treatment for the current diagnosis until documented treatment failure or progression. Freedom from failure and overall survival rates were calculated using the Kaplan-Meier method $^{21}$. A log-rank test was used for univariate analysis to compare the differences of the survival probabilities. Multivariate analysis using a Cox regression model was performed to define significant prognostic factors. All analyses were performed in SPSS statistics version 18.0 software package (Chicago, IL USA).

Acute adverse events were scored using the Common Terminology Criteria for Adverse Events version (CTC AE) version 4.03, and included the adverse events that occurred during or within 3 months after the initiation of CIRT. Late toxicities were scored using the Radiation Therapy Oncology Group (RTOG) late radiation morbidity scoring system for toxicities observed beginning at 90 days after completion of CIRT.

\section{Results}

\section{Characteristics of patients, their tumors, and initial treatment}

Nineteen (19) consecutive and non-selected patients with recurrent HNS treated with IMCT alone (16 patients) or a combination of proton and CIRT (3 patients) with a curative intent between October 2015

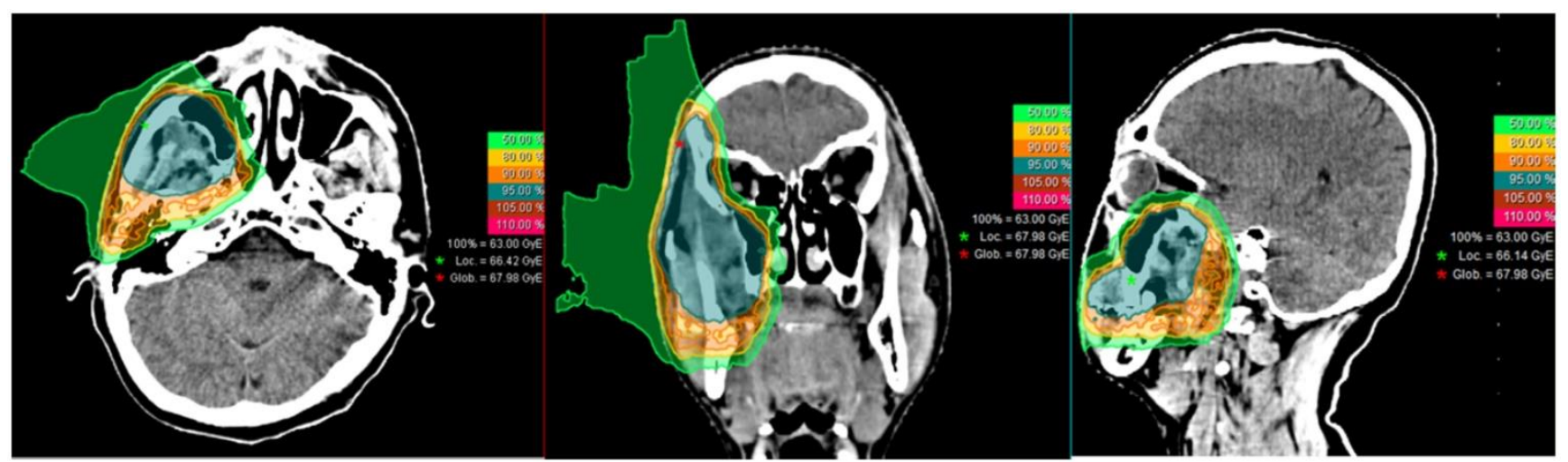

Figure 1. Typical treatment re-irradiation plan of a patient with a locally recurrent soft-tissue sarcoma of the buccal region. 
and July 2017 at SPHIC were analyzed. No patients with recurrent HNS were excluded from this analysis. The median follow-up was 13.1 (range 1.57-41.10) months for the entire cohort.

Eight (8) patients (including 6 chondrosarcoma and 2 STS) presented with locally recurrent HNS after surgery without prior radiation therapy. Eleven patients had one prior course of head and neck radiotherapy to a definitive dose (median $68 \mathrm{~Gy}$, range 13-78 Gy), with or without surgery. Among these 11 patients, 4 had previous RT, either definitively or adjuvantly, for STS and were then re-irradiated with IMCT for a local recurrence; 7 patients had photon-based RT for undifferentiated nasopharyngeal carcinoma (NPC) (4 cases, intensity-modulated photon radiotherapy, i.e., IMXT), poorly differentiated squamous cell cancer (SCC) of the ethmoid sinus ( 1 case, IMXT), base of the skull chordoma (1 case, IMXT), or a pituitary adenoma (1 case, GammaKnife) then subsequently developed an RT-induced second primary STS (3 cases), osteosarcoma (2 cases), small round cell sarcoma (1 case), or chondrosarcoma (1 case) at the previous disease/irradiated site.

The interval between the current course of IMCT and their prior RT for all 11 re-irradiated patients was $>12$ months and the median time was 95.24 months (range 12.30-169.40). The characteristics of patients and their conditions, as well as their prior treatment for primary diseases, are detailed in Table 1 and Table 2 , respectively.

\section{Carbon ion radiation therapy and adjuvant treatment}

All patients received intensity-modulated CIRT using pencil beam scanning technology including 15 that completed salvage IMCT alone and 3 completed a combination of proton and IMCT boost. One patient discontinued IMCT after 4 fractions due to Grade 4 bleeding then a stroke caused by embolization of the internal carotid artery. The median clinical target volume (CTV) for gross tumor volume (CTV-G) was 85.87 Cc (range 32.90-212.48) and the median D95 of the CTV-G was 58.10 GyE (range 47.90-64.12). The summary of PRT is shown in Table 3.

Four patients $(21.1 \%)$ with soft-tissue sarcoma or osteosarcoma also received chemotherapy. Nine patients $(47.4 \%)$ (5 with soft-tissue sarcoma and 4 with chondrosarcoma) had a salvage surgery prior to CIRT.

\section{Overall survival}

The actuarial 12-month OS was $86.5 \%$ (Figure 2c) for the entire group. Three of the 7 patients with a second primary sarcoma who received salvage CIRT were deceased at the time of this analysis. One died of local progression at 11.9 months after CIRT for a second primary small round cell sarcoma after previous RT for pituitary adenoma. Two other patients with secondary osteosarcoma or undifferentiated sarcoma after definitive IMXT for NPC died of local progression or massive bleeding of the internal carotid artery at 5.6 and 3.3 months after IMCT reirradiation, respectively.

Table 1. Characteristics of the patients and their conditions

\begin{tabular}{|c|c|c|}
\hline Characteristic & No. & $\%$ \\
\hline Age median (range) & \multicolumn{2}{|c|}{44 (19-68) years } \\
\hline \multicolumn{3}{|l|}{ Sex } \\
\hline Male & 11 & 57.9 \\
\hline Female & 8 & 42.1 \\
\hline \multicolumn{3}{|l|}{ KPS } \\
\hline 80 & 4 & 21.0 \\
\hline 90 & 15 & 79.0 \\
\hline \multicolumn{3}{|l|}{ Histology } \\
\hline Chondrosarcoma & 7 & 36.8 \\
\hline Rhabdomyosarcoma & 3 & 15.9 \\
\hline Undifferentiated/unclassified & 2 & 10.5 \\
\hline Spindle cell sarcoma & 1 & 5.3 \\
\hline Pleomorphic sarcoma & 1 & 5.3 \\
\hline Small round cell sarcoma & 1 & 5.3 \\
\hline Desmoid-type fibromatosis & 1 & 5.3 \\
\hline Synovial sarcoma & 1 & 5.3 \\
\hline Osteosarcoma & 2 & 10.5 \\
\hline \multicolumn{3}{|l|}{ Site } \\
\hline Skull base & 8 & 42.1 \\
\hline Nasal cavity-paranasal sinus & 7 & 36.8 \\
\hline Others & 4 & 21.0 \\
\hline \multicolumn{3}{|l|}{ Second primary } \\
\hline Yes (second primary) & 7 & 36.8 \\
\hline No (locally recurrent) & 12 & 63.2 \\
\hline \multicolumn{3}{|l|}{ Previous radiotherapy } \\
\hline Yes & 11 & 57.9 \\
\hline No & 8 & 42.1 \\
\hline \multicolumn{3}{|l|}{ Smoking } \\
\hline Yes & 3 & 15.8 \\
\hline No & 16 & 84.2 \\
\hline
\end{tabular}

Table 2. Summary of characteristics of patients with prior radiotherapy

\begin{tabular}{llll}
\hline & All & $\begin{array}{l}\text { Soft tissue and } \\
\text { bone sarcoma }\end{array}$ & Chondrosarcoma \\
\hline $\begin{array}{l}\text { Previous radiation dose } \\
\text { (Gy) }\end{array}$ & $\begin{array}{l}\text { Median } \\
68(13-78)\end{array}$ & $\begin{array}{l}\text { Median } \\
63(13-78)\end{array}$ & $\begin{array}{l}\text { Median } \\
75.35 \text { (1 patient) }\end{array}$ \\
$\begin{array}{l}\text { Interval between last } \\
\text { treatment and this } \\
\text { treatment (months) }\end{array}$ & 30.63 & 48.40 & 22.97 (1 patient) \\
$\begin{array}{l}\text { Interval between last RT } \\
\text { and re-RT (months) }\end{array}$ & $(2.57-169.40)$ & $(2.57-169.4)$ & \\
\hline
\end{tabular}

Table 3. Summary of the salvage particle beam treatment for all patients

\begin{tabular}{llll}
\hline & All N (\%) & $\begin{array}{l}\text { Soft tissue and bone } \\
\text { sarcoma }\end{array}$ & Chondrosarcoma \\
\hline IMCT & 16 & 11 & 5 \\
IMPT + IMCT Boost & 3 & 1 & 2 \\
Median no. of fields & 3 & 3 & 3 \\
(range) & $(1-4)$ & $(1-3)$ & $(1-4)$ \\
Median CTV-G volume & 85.87 & 93.01 & 75.96 \\
in cc (range) & $(32.90-212.48)$ & $(32.90-212.48)$ & $(42.01-212.35)$ \\
Median CTV-G D95 in & 58.10 & 58.10 & 57.96 \\
GyE (range) & $(47.90-64.12)$ & $(47.90-61.94)$ & $(53.59-64.12)$ \\
\hline
\end{tabular}




\section{Local and distant failure- and progression-free survival}

Two patients with rhabdomyosarcoma and 1 patient with chondrosarcoma developed distant metastasis after the completion of CIRT at 4.76, 2.0, and 0.33 months, respectively. Two patients with second primary STS/osteosarcoma as mentioned above and another with spindle cell sarcoma developed local progression at 3.76, 8.51 and 10.17 months, respectively, after CIRT. Five of these 6 patients with treatment failure (local or distant) had received prior photon-based radiation. The 12-month cumulative local progression free survival (LPFS) rate was $74.6 \%$ (Figure 2a). The 12-month distant metastasis free survival (DMFS) rate was 82.6\% (Figure 2b). The progression-free survival (PFS) at 12 months was 53.6\% (Figure 2d).

\section{Prognostic Factors}

Univariate analysis showed that OS was associated with the presence of a second primary tumor $(p=0.008)$, and disease-progression was associated with tumor site $(\mathrm{p}=0.038)$. In addition, there was a trend that local-regional relapse and
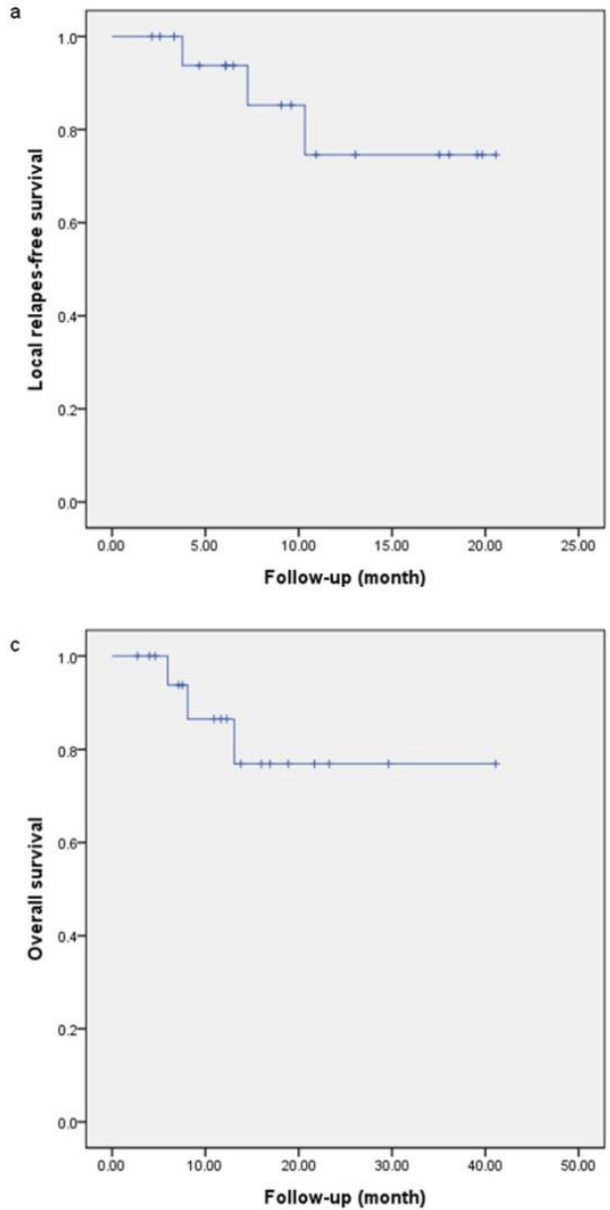

disease-progression were associated with re-radiotherapy ( $p=0.086,0.076$, respectively).

Multivariate analysis was performed to examine the impact of various prognostic factors, including sex (male vs. female), status of RT (re-RT vs. first-time RT), second primary tumor (RT-induced second primary vs. locally recurrent tumor), tumor site (skull base vs. sinonasal vs. others), histology (STS/osteosarcoma vs. chondrosarcoma), particle radiotherapy (CIRT vs. proton + CIRT boost), surgery (with vs. without), chemotherapy (with vs. without), and treatment pattern (surgery + particle radiotherapy [PRT] vs. PRT alone vs. surgery + chemotherapy + PRT vs. chemotherapy + PRT) for predicting patients' prognoses. The 1-year LRFS, DMFS, OS, and PFS rates were evaluated respectively as endpoints. No independent prognostic factor was identified for OS, PFS, LFFS, or DMFS. However, a trend was found for second primary tumors in independently predicting PFS ( $\mathrm{p}=0.055$, Table 4$)$.

\section{Acute and late toxicities}

Eighteen (18) patients completed their planned treatment. One patient prematurely discontinued
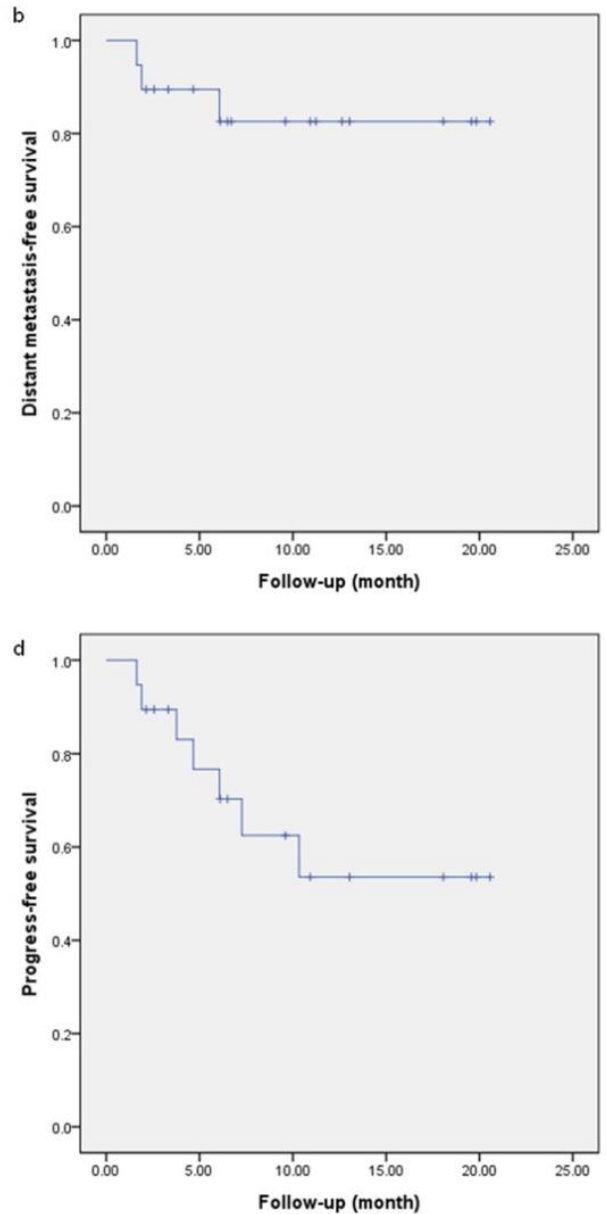

Figure 2. Local progression-free survival (a), distant metastatic-free survival (b), overall survival (c), and progression-free survival (d) curves of the entire cohort. 
after 4 fractions of IMCT (received a total dose of 12 GyE) due to bleeding at the disease site from rupturing of an optic artery aneurysm. The hemorrhage was confirmed to be unrelated to his disease and treatment. The bleeding aneurysm was confirmed on an angiogram during embolization and a minimal CIRT dose was delivered.

Table 4. Cox proportional hazard regression analysis for 1-year PFS

\begin{tabular}{llllll}
\hline & Wald & Sig. & HR & \multicolumn{2}{c}{$95.0 \%$ CI for Exp(B) } \\
\cline { 5 - 6 } & & & & Lower & Upper \\
\hline Gender & 0.262 & 0.609 & 2.380 & 0.086 & 65.884 \\
Re-radiotherapy & 2.345 & 0.126 & 69.159 & 0.306 & 15651.325 \\
Second primary & 3.685 & $\mathbf{0 . 0 5 5}$ & 0.009 & 0.000 & 1.105 \\
Site & 0.314 & 0.575 & 1.920 & 0.196 & 18.802 \\
Histology & 0.396 & 0.529 & 3.327 & 0.079 & 140.499 \\
PRT & 0.001 & 0.978 & 0.000 & 0.000 & $<0.0001$ \\
Surgery & 0.891 & 0.345 & 0.107 & 0.001 & 11.121 \\
Chemotherapy & 2.026 & 0.155 & 97895.108 & 0.013 & $7.289 \mathrm{E} 11$ \\
Treatment pattern & 2.955 & 0.086 & 0.002 & 0.000 & 2.335 \\
Abbreviations: PRT, particle radiotherapy; PFS, progress-free survival; CI = \\
\multicolumn{2}{l}{ confidence interval; HR = hazard ratio. }
\end{tabular}

Table 5. Type and frequency of acute adverse events

\begin{tabular}{|c|c|c|c|c|c|c|c|c|}
\hline \multirow[t]{3}{*}{ Toxicity } & \multicolumn{8}{|c|}{ Grade } \\
\hline & \multicolumn{2}{|c|}{1} & \multicolumn{2}{|l|}{2} & \multicolumn{2}{|l|}{3} & \multicolumn{2}{|l|}{4} \\
\hline & No. & $\%$ & No. & $\%$ & No. & $\%$ & No. & $\%$ \\
\hline $\begin{array}{l}\text { Mucous } \\
\text { membrane }\end{array}$ & 2 & 10.5 & 2 & 10.5 & 1 & 5.3 & 0 & 0 \\
\hline Skin & 7 & 36.8 & 0 & 0 & 0 & 0 & 0 & 0 \\
\hline Hemorrhage & 1 & 5.3 & 0 & 0 & 0 & 0 & 2 & $10.5^{*}$ \\
\hline
\end{tabular}

* Including one case of hemorrhage due to a confirmed rupturing of an inter-concurrent optic artery aneurysm unrelated to the disease or treatment confirmed on angiogram.

Table 6. Type and frequency of late toxicities

\begin{tabular}{|c|c|c|c|c|c|c|c|c|c|c|}
\hline \multirow[t]{3}{*}{ Toxicity } & \multicolumn{10}{|c|}{ Grade } \\
\hline & \multicolumn{2}{|c|}{1} & \multicolumn{2}{|l|}{2} & \multicolumn{2}{|l|}{3} & \multicolumn{2}{|l|}{4} & \multicolumn{2}{|l|}{5} \\
\hline & No. & $\%$ & No. & $\%$ & No. & $\%$ & No. & $\%$ & No. & $\%$ \\
\hline Headache & 1 & 5.3 & 0 & 0 & 0 & 0 & 0 & 0 & 0 & 0 \\
\hline Dry mouth & 1 & 5.3 & 0 & 0 & 0 & 0 & 0 & 0 & 0 & 0 \\
\hline Hemorrhage & 0 & 0 & 0 & 0 & 0 & 0 & 0 & 0 & 1 & $5.3^{*}$ \\
\hline Pain & 1 & 5.3 & 0 & 0 & 0 & 0 & 0 & 0 & 0 & 0 \\
\hline Skin reaction & 1 & 5.3 & 0 & 0 & 0 & 0 & 0 & 0 & 0 & 0 \\
\hline
\end{tabular}

* Same patient who had Grade 4 acute hemorrhage of the sphenopalatine artery.

Another patient with a radiation-induced second primary sarcoma from IMRT (68 Gy in 30 fractions) with concurrent chemotherapy for locally advanced NPC 11 years ago experienced Grade 4 acute hemorrhage from the sphenopalatine artery during salvage CIRT. The bleeding was salvaged by embolism. The patient received resection of the tumor in the post-nasal space 1 month before the initiation of salvage CIRT, then completed salvage CIRT to $60 \mathrm{GyE}$ (in 20 fractions). As the bleeding sphenopalatine artery was encompassed in both the initial and salvage radiation fields, this adverse event of Grade 4 hemorrhage was defined to be radiation-induced. The same patient later died from severe (Grade 5) hemorrhage 3.5 months after the completion of re-irradiation. The exact site of the bleeding was not determined due to the speed of the death. The maximum and mean doses from the salvage CIRT to the left/right carotid arteries were 61.21/61.22 GyE and 56.52/51.86 GyE (in 20 fractions). Unfortunately, the doses to the carotid arteries from the initial IMRT were not available to us, although it was likely that the full dose of 68 Gy from the initial IMRT covered both arteries for the locally advanced NPC. Nevertheless, bleeding from a carotid blowout could not be ruled out and was likely the cause of his fatal hemorrhage.

The types and severity of acute and late toxicities are detailed in Tables 5 and 6.

\section{Discussion}

In this study, we have analyzed 19 consecutive and non-selected patients with locally recurrent or radiation-induced second primary HNS treated with intensity-modulated carbon ion radiation therapy (IMCT) using pencil beam scanning (PBS) technology. We have reported a relatively favorable 1-year survival of $86.5 \%$ for this challenging condition. Three patients with radiation-induced second primary sarcoma died after CIRT reirradiation: 2 from local progression and 1 from hemorrhage. One additional patient experienced local progression after CIRT. The 1-year LPFS rate approached $75 \%$. Two patients experienced Grade 4 hemorrhage during treatment, including one from an unrelated rupture of an optic artery aneurysm. Another patient developed radiation-induced Grade 4 hemorrhage of the sphenopalatine artery, and later died of Grade 5 hemorrhage 3.5 months after the completion of CIRT. Otherwise, no Grade 3 or Grade 4 acute or late toxicities were observed.

Radiation therapy (RT) is one of the most important components of the multi-modality management of HNS. Complete surgical resection is difficult to achieve for HNS and adjuvant RT is usually necessary after R1 or R2 surgery. Due to the complexity of the anatomy, doses of RT can be limited due to the proximity to the critical organs at risk (OARs). The physical characteristics of PRT make it particularly suitable for treating malignancies situated close to the OARs. A Bragg peak is formed when the energy of a charged particle is precisely deposited at the designated depth followed by a steep dose reduction. PRT is the preferred technology to treat base of the skull chordomas, chondrosarcomas, and other deeply located head and neck tumors, especially those within the vicinity of vulnerable tissues $^{22,23}$. Furthermore, carbon ion is a high-LET radiation, and the value of relative biological 
effectiveness (RBE) of CIRT is 3 5, depending on the tumor or tissue type as well as the end point of the study. As such, CIRT is theorized to be more effective in disease control of more radio-resistant tumors including many types of sarcomas, especially for those patients who did not respond to photon-based RT.

The clinical outcomes of sarcomas treated with CIRT have been reported in a number of publications. In a retrospective study reported from the National Institute of Radiation Science (NIRS), 24 patients with retroperitoneal sarcoma were treated with 52.8 to 73.6 GyE of CIRT in 16 fixed fractions over 4 weeks ${ }^{24}$. The 2 -year OS and LC rates were $75 \%$ and $77 \%$, respectively. No severe GI complications were observed. In a phase I/II trial, Sugahara et al. reported the results of CIRT in the treatment of 17 patients with soft-tissue sarcoma of the extremities, including 8 with recurrent disease after surgery with or without chemotherapy ${ }^{25}$. None of the 17 patients had prior radiation therapy. Mixed dose/fraction dose escalation schemes were used in the trial, with the highest dose/fraction at 70.4 GyE (4.4 GyE/fraction). The authors reported 3-year OS and local control rates of $68 \%$ and $76 \%$, respectively. No Grade 4 acute or late toxicity was observed. Similar results were reported for 47 patients with primary spinal sarcoma after CIRT $^{26}$. The 5-year LC and OS rates were $79 \%$ and $52 \%$, respectively, after CIRT to a median dose of 60.4 GyE in 16 fractions. Furthermore, the use of CIRT is reportedly safe and effective for primary skull base chondrosarcomas treated with CIRT to $60 \mathrm{GyE}$ (3 $\mathrm{GyE} /$ fraction). The reported 3-year LC and OS rates were $95.9 \%$ and $96.1 \%$, respectively, in a study with 79 patients irradiated using CIRT27. The 1-year LC and OS were equally favorable. In addition, no radiation-induced secondary malignancies were observed.

Despite the favorable outcomes after CIRT for sarcomas of the trunk, literature on the use of CIRT in the treatment of recurrent or radiation-induced second primary sarcomas in the head and neck region is lacking. A thorough literature search only resulted in case reports of locally recurrent HNS treated with CIRT ${ }^{28}$. Therefore, a meaningful analysis is not possible to determine its efficacy. However, the physical and biological characteristics make CIRT an ideal radiation modality for cancers that have failed to respond to previous radiation therapy for two reasons: 1) The heavily irradiated OARs from prior RT can be effectively excluded from high dose re-irradiation using CIRT. 2) Local recurrences after prior RT are typically more resistant to repeated photon-based irradiation. CIRT offers a higher RBE, which is critical in disease control in such a clinical scenario. Salvage treatment using CIRT for nasopharyngeal cancer (NPC) that failed to respond to previous high-dose radiation is currently being studied in 2 prospective clinical trials at our center ${ }^{18,29}$. Preliminary results have indicated the safety and effectiveness of such a regimen ${ }^{30}$.

Based on our literature search, the use of CIRT for the management of locally recurrent or second primary soft-tissue and osteosarcoma has never been addressed. Reports on the use of CIRT for recurrent base of the skull chondrosarcoma are scarce and are usually combined with non-sarcomatous pathology. Nevertheless, despite the novelty of our study, this paper is limited by a number of shortcomings. First, the number of patients in this cohort is small. Due to the complexity of the disease, the pathology subtypes are mixed. Among the 19 patients, 7 patients had a radiation-induced second primary HNS, 4 had locally recurrent HNS after prior photon-based radiation, and 8 had recurrences after definitive surgery without prior radiation. In fact, the literature on re-irradiation (photon beam) for HNS sarcoma is extremely limited as well. A limited number of case reports or series have indicated that such a practice is associated with severe adverse effects, and it may only provide palliative effects in the majority of patients ${ }^{31}$. Given the rarity of the condition and the novelty of the CIRT technology, it is unlikely that prospective trials or large-scale retrospective series would be available on this topic in the near future. Although treatment strategies for different histologies and stages are different for primary HNS, regimens for effective control of HNS are limited after prior aggressive multimodality treatment. The histology subtypes of the 19 patients in our series are mixed, but all had previously undergone surgery and/or radiotherapy for their primary disease and were treated in a relatively uniform fashion with CIRT, although 3 patients received proton RT followed by a CIRT boost. Second, the follow-up time is relatively short for our patients. Nonetheless, given the acknowledged poor prognosis in recurrent or second primary HNS $32,33,34$, and the fact that the majority of treatment failures for nearly all head and neck cancers occurs within the first 2 years, outcomes in terms of toxicities, local control, and overall survival with a median follow-up time of 14 months remains valuable to understand the efficacy and safety of CIRT in such conditions.

The overall outcome of this patient cohort is encouraging. Based on our data and experience, a phase 2 prospective clinical trial is being designed for CIRT re-irradiation in the management of patients with locally recurrent HNS, particularly those with previous photon-based irradiation, to determine the efficacy of salvage CIRT. For patients with 
radiation-induced second primary sarcoma, a more effective treatment strategy is obviously needed. Since 2 of 7 patients developed grade 4 hemorrhage during salvage CIRT, we now encourage all patients to be evaluated by a vascular surgeon and embolization of any major artery at risk is the current standard recommendation before the initiation of CIRT reirradiation.

\section{Conclusions}

Salvage CIRT used in patients with locally recurrent HNS with or without previous radiotherapy produced a relatively favorable 1-year outcome with an OS of $100 \%$ without moderate or severe acute or late adverse effects. Although such a practice may also be valuable for managing patients with radiation-induced second primary sarcoma, hemorrhage secondary to tumor progression or re-irradiation might be a significant hindering factor for tumors in the head and neck area. A more aggressive multi-modality strategy is also needed to improve local control in patients with second primary HNS.

\section{Acknowledgments}

\section{Funding Source(s):}

Science and Technology Commission of Shanghai Municipality (Project No. 15411950102 \& 15411950106)

Shanghai Municipal Commission of Health and Family Planning (Project No. 20174Y0076)

Joint Breakthrough Project for New Frontier Technologies of the Shanghai Hospital Development Center (Project No. SHDC22017101)

\section{Competing Interests}

The authors have declared that no competing interest exists.

\section{References}

1 Farhood AI, Hajdu SI, Shiu MH, et al. Soft tissue sarcomas of the head and neck in adults. Am J Surg 1990;160:365-369.

2 Chen SA, Morris CG, Amdur RJ, et al. Adult head and neck soft tissue sarcomas. Am J Clin Oncol 2005;28:259-263.

3 Mendenhall WM, Mendenhall CM, Werning JW, et al. Adult head and neck soft tissue sarcomas. Head Neck 2005;27:916-922.

4 Eeles RA, Fisher C, A'Hern RP, et al. Head and neck sarcomas: Prognostic factors and implications for treatment. $\mathrm{Br} \mathrm{J}$ Cancer 1993;68:201-207.

5 Huber GF, Matthews TW, Dort JC. Soft-tissue sarcomas of the head and neck: A retrospective analysis of the Alberta experience 1974 to 1999. Laryngoscope 2006;116:780-785.

6 Ballo MT, Zagars GK, Cormier JN, et al. Interval between surgery and radiotherapy: Effect on local control of soft tissue sarcoma. Int J Radiat Oncol Biol Phys 2004;58:1461-1467.

7 Gil Z, Patel SG, Singh B, et al. Analysis of prognostic factors in 146 patients with anterior skull base sarcoma: An international collaborative study. Cancer 2007;110:1033-1041.
8 Pisters PW, Harrison LB, Leung DH, et al. Long-term results of a prospective randomized trial of adjuvant brachytherapy in soft tissue sarcoma. J Clin Oncol 1996;14:859-868.

9 Yang JC, Chang AE, Baker AR, et al. Randomized prospective study of the benefit of adjuvant radiation therapy in the treatment of soft tissue sarcomas of the extremity. J Clin Oncol 1998;16:197-203.

10 O'Sullivan B, Davis AM, Turcotte R, et al. Preoperative versus postoperative radiotherapy in soft-tissue sarcoma of the limbs: A randomized trial. Lancet 2002;29:2235-2241.

11 Davis AM, O'Sullivan B, Turcotte $\mathrm{R}$, et al. Late radiation morbidity following randomization to preoperative versus postoperative radiotherapy in extremity soft tissue sarcoma. Radiother Oncol 2005;75:48-53.

12 Kassir RR, Rassekh CH, Kinsella JB, et al. Osteosarcoma of the head and neck: meta-analysis of nonrandomized studies. Laryngoscope 1997;107:56-61.

13 Kepka L, DeLaney TF, Suit HD, et al. Results of radiation therapy for unresected soft-tissue sarcomas. Int J Radiat Oncol Biol Phys 2005;63:852-859.

14 Tsujii H, Kamada T, Baba M, et al. Clinical advantages of carbon-ion radiotherapy. New J Phys 2008;10:075009.

15 Kanai T, Endo M, Minohara S, et al. Biophysical characteristics of HIMAC clinical irradiation system for heavy-ion radiation therapy. Int J Radiat Oncol Biol Phys 1999;44: 201-210.

16 Kong L, Lu JJ. Reirradiation of locally recurrent nasopharyngeal cancer: history, advances, and promises for the future. Chin Clin Oncol. 2016 Apr;5(2):26. doi: 10.21037/cco.2016.03.19.

17 Le ZY, Liu Y. The Research Progress in the Radiation Biology of Heavy Ion. Chinese Journal of Radiation Oncology, 2015, 24(5): 600-604.

18 Kong L, Hu J, Guan X, Gao J, Lu R, Lu JJ. Phase I/II Trial Evaluating Carbon Ion Radiotherapy for Salvaging Treatment of Locally Recurrent Nasopharyngeal Carcinoma. J Cancer. 2016 Apr 10;7(7):774-83. doi: 10.7150/jca.14399.

19 Nieder C, Milas L, Ang KK. Tissue tolerance to reirradiation. Semin Radiat Oncol. 2000 Jul;10(3):200-9.

20 Koto M. Skull base and upper cervical spine tumors. In: Tsujii $\mathrm{H}$, Kamada T, Shirai T, et al, Eds. In Carbon-Ion Radiotherapy Principles, Practices, and Treatment Planning. Heidelberg: Springer. 2014: 155-161.

21 Kaplan EL, Meier P. Nonparametric estimation from incomplete observations. Journal of the American Statistical Association. 1958;53:25.

22 Noel G, Gondi V. Proton therapy for tumors of the base of the skull. Chin Clin Oncol 2016;5(4):51. doi: 10.21037/cco.2016.07.05

23 ASTRO. Proton beam therapy (PBT). Available at: https://www.astro.org/uploadedFiles/Main_Site/Practice_Manageme nt/Reimbursement/ASTRO\%20PBT\%20Model\%20Policy\%20FINAL.pd f [Accessed August 10, 2017].

24 Serizawa I, Kagei K, Kamada T, Imai R, Sugahara S, Okada T, et al. Carbon ion radiotherapy for unresectable retroperitoneal sarcomas. Int J Radiat Oncol Biol Phys. 2009 Nov 15;75(4):1105-10. doi: 10.1016/j.ijrobp.2008.12.019.

25 Sugahara S, Kamada T, Imai R, Tsuji H, Kameda N, Okada T, et al. Carbon ion radiotherapy for localized primary sarcoma of the extremities: results of a phase I/II trial. Radiother Oncol. 2012 Nov;105(2):226-31. doi: 10.1016/j.radonc.2012.09.010.

26 Matsumoto K, Imai R, Kamada T, Maruyama K, Tsuji H, Tsujii H, et al. Impact of carbon ion radiotherapy for primary spinal sarcoma. Cancer. 2013 Oct 1;119(19):3496-503. doi: 10.1002/cncr.28177.

27 Uhl M, Mattke M, Welzel T, Oelmann J, Habl G, Jensen AD, et al. High control rate in patients with chondrosarcoma of the skull base after carbon ion therapy: first report of long-term results. Cancer. 2014 May 15;120(10):1579-85. doi: 10.1002/cncr.28606.

28 Combs SE, Kalbe A, Nikoghosyan A, Ackermann B, Jäkel O, Haberer T, Debus J. Carbon ion radiotherapy performed as re-irradiation using active beam delivery in patients with tumors of the brain, skull base and sacral region. Radiother Oncol. 2011 Jan;98(1):63-7. doi: 10.1016/j.radonc.2010.10.010

29 Kong L, Gao J, Hu J, Hu W, Guan X, Lu R, Lu JJ. Phase I/II trial evaluating concurrent carbon-ion radiotherapy plus chemotherapy for salvage treatment of locally recurrent nasopharyngeal carcinoma. Chin J Cancer. 2016 Dec 22;35(1):101. doi: 10.1186/s40880-016-0164-5.

30 Kong L, Wang L, Guan X, et al. Efficacy and Toxicity of Intensity Modulated Carbon Ion Radiation Therapy for Local Recurrent Nasopharyngeal Cancer. Int J Radiat Oncol Biol Phys 2016;96(2) Supplement: E353-E354

31 Graham JD, Robinson MH, Harmer CL. Re-irradiation of soft-tissue sarcoma. Br J Radiol. 1992 Feb;65(770):157-61. 
32 Riad S, Biau D, Holt GE, et al. The clinical and functional outcome for patients with radiation-induced soft tissue sarcoma. Cancer 2012; 118:2682.

33 Patel SG, See AC, Williamson PA, et al. Radiation induced sarcoma of the head and neck. Head Neck 1999; 21:346.

34 Yeang MS, Tay K, Ong WS, et al. Outcomes and prognostic factors of post-irradiation and de novo sarcomas of the head and neck: a histologically matched case-control study. Ann Surg Oncol 2013; 20:3066. 